\title{
TOO BIG TO HEAL? MACROPRUDENTIAL POLICIES IN HEALTH GOVERNANCE
}

\author{
Henrique Choer Moraes ${ }^{1}$
}

\begin{abstract}
Published at Global Policy website, 29 April 2020
(https://www.globalpolicyjournal.com/blog/29/04/2020/too-big-heal-macroprudentialpolicies-health-governance)
\end{abstract}

Amidst the many consequences it will leave in its wake, COVID-19 might reveal that global health governance should focus on systemic risk management, just as their peers in financial regulation did over a decade ago.

The emergence of a pandemic such as COVID-19 has been expected for quite some time. To take a recent example, the September 2019 annual report by the Global Preparedness Monitoring Board alerted about "a rapidly spreading, lethal respiratory pathogen pandemic". Yet, when this report spelled out the progress indicators in tackling such pandemic, it tellingly overlooked what is proving to be the immediate roadblocks in the struggle against the pandemic, namely the shortage of personal protective equipment (PPE), ventilators and testing kits.

What is gravely symptomatic, though, is that global health mechanisms were left to reactinstead of alert in advance - to the effects of the structural bottlenecks in the supply chains of such medical assets. It was on March $3^{\text {rd- }}$ in the intervening period between characterizing the virus as a public health emergency of international concern (January $30^{\text {th }}$ ) and a pandemic (March $11^{\text {th }}$ ) - that the WHO was calling on "industry and governments to increase manufacturing [of PPEs] by 40 per cent to meet rising global demand".

Could we have arrived at this moment better prepared? Should global health governance have been addressing systemic-level features such as the supply chains of resources like PPE before the outbreak of a pandemic?

Throughout its history, the WHO has developed expertise in risk management, including experience of almost 70 successful years in bringing together a network of surveillance and response to influenza on a world scale. While this pool of resources has performed well in tackling illnesses such as the 2003 SARS, the outbreak of COVID-19 poses a systemic risk for which health governance might not be fully equipped to address.

Faced with the equally disruptive effects of the 2008 crash, global financial governance took precisely the step of reengineering its regulatory approach, from firm-level risk managementproved ineffective by the crisis - to systemic-level regulation by means of a number of macroprudential policies.

\section{FROM "TOO BIG TO FAIL" TO "TOO BIG TO HEAL"}

\footnotetext{
${ }^{1}$ All views and opinions in this article are the sole responsibility of the author and do not necessarily reflect the positions of the government of Brazil.
} 
The global financial crisis was a wake-up call for regulators to the importance of focusing on the broader structure of the financial system, particularly the degree of interconnectedness between its components as well as the level of risk generated by the sheer size of some of them, those considered "too big to fail". As a former general manager of the Bank for International Settlements put it in 2010, regulators "were faced with the unthinkable when a number of very large institutions failed, despite their previous reputation for balance sheet strength and leadership in risk management”.

As a reaction to the regulatory shortcomings made clear by the 2008 financial crisis, policymakers developed a number of macroprudential tools aimed at better spotting vulnerabilities that compromise the resilience of the system in the event of disruption, such as the bankruptcy of a highly interconnected financial institution. One of these tools is the regulation of "global systemically important banks" (G-SIBs), a technical name to label those units in the system considered "too big to fail" - and which are since then subject to stricter rules at both national and international levels. Every year, a list of the G-SIBs is prepared following indicators that assess banks' size, interconnectedness, substitutability, complexity and cross-border activity.

Given the risks they present to the stability of the financial system in case of a disruption, GSIBs are subject to stricter rules in areas such as resolution, capitalization and monitoring. Underlying the concept of G-SIBs is the notion that certain elements in the system have such a level of centrality that they warrant reinforced regulatory attention in order to preserve the system itself.

Other macroprudential financial policies have sought to strengthen the resilience of the financial system at the global and national levels. They include frameworks for monitoring systemic risks; early warning indicators that signal emerging vulnerabilities in the financial system; and measures to reduce contagion, such as increasing the capacity of G-SIBs to absorb losses as well as limiting exposure within the financial system.

Whereas the financial and health regimes have significant differences - and thus solutions in one area might not be directly suitable to the other-, the regulatory leap undertaken by financial regulators after 2008 can contribute to the debates that will take place in the postCOVID world.

Just as the collapse of "too big to fail" banks magnify the impact of vulnerabilities existing in the financial system, are there global health challenges whose emergence can bring to light systemic failures that undermine resilience in health, such as bottlenecks in supply chains of medical equipment? Are there diseases too big to heal? Or can preventive action be implemented in mitigating systemic risks?

\section{MACROPRUDENTIAL HEALTH MEASURES}

When it comes to health governance, it is reasonable to argue that some challenges can prove to be systemically important so as to require strengthened attention at the national and international levels. COVID-19 could rank as a "global systemically important disease" (GSID) of sorts. 
Macroprudential health policies would focus on preventive action viewing the health system as a whole. Increased attention by regulators to systemic risks would include measures such as the following:

(i) identification of the G-SIDs which should be subject to upgraded systemic risk management measures;

(ii) monitoring the build-up of risks that can contribute to outbreaks of G-SIDs. This would focus on practices that favor the spread of G-SIDs, such as, for example the persistence of wet markets, in the case of COVID-19, but also of other infectious diseases;

(iii) mapping the key nodes in the system that need support in the event of a G-SID. This would include, for example, mapping out the structure of global supply chains of treatments and medical equipment required to fight a G-SID, and proposing appropriate action at the national and international levels to increase resilience in cases of disruption.

The latter example illustrates the importance of a macroprudential approach to health risks.

The WHO has a commendable record for increasing risk management across the world. Understandably, the focus of this work is directed at national preparedness. For example, the WHO document A checklist for pandemic influenza risk and impact management - 2018 update recommends countries to "[c]onsider securing access to antiviral drugs, pandemic vaccines, diagnostics and other products". This recommendation is a sensible risk management proposition from the individual perspective of each country.

But what happens when all countries decide to "secure access" to these resources at the same time?

The experience with COVID-19 reveals that systemic health risks emerge from chokeholds in the supply of medical equipment stemming from over-reliance on a small and geographically concentrated number of providers. These risks are not captured when the unit of risk management is the country.

Certainly it would have been virtually impossible to predict the moment and volume of the surge in the demand for such resources as PPE. Still, considering that the outbreak of a pandemic was being expected any time soon, would it not be warranted for the global health governance mechanisms to monitor the structure of global supply chains of key resources such as PPE, thereby assessing systemic risks that might arise from vulnerabilities in such structure?

To be sure, it might be far-fetched to consider entirely nationalizing supply chains. At the same time, COVID-19 challenges the current structure of global supply chains, which have been designed predominantly by a market-oriented logic of efficiency. Such challenge seems particularly valid at least with respect to essential goods such as those deployed in combating a pandemic. It is between these two extremes that macroprudential health measures could be discussed to address this systemic risk.

\section{CONCLUSION - A NEW ROLE FOR G20 IN HEALTH?}

Many lessons will be drawn in the wake of the coronavirus pandemic. 
One possibility is that the virus accelerates the movement toward geopolitical disputes, which might lead to outbursts of "health nationalisms" marked by autarky, including in the development of treatment.

Another scenario is that we learn from the experience we are going through and use it to improve solutions at the global and national levels. For all the positive record of the WHO in helping countries to prepare and respond to pandemics, the outbreak of coronavirus sheds light on systemic aspects of global health challenges that do not seem to be addressed by the existing governance mechanisms. Despite the many differences with the 2008 crisis, the "macroprudential turn" adopted by regulators in the financial realm might inspire the search for solutions in health governance. Increased attention by health regulators to systemic risks would, similarly to the experience in financial governance, be a move in the direction of resilience of the system.

Another possible parallel to the developments following the global financial crisis could emerge from the institutional side. Post-COVID health governance could see an increased role for the $\mathrm{G} 20$ in coordinating the work of the WHO with that of other international regimes just as the G20 steered the overhaul of global financial governance by coordinating the work of agencies as the IMF, BCBS and the then newly-established Financial Stability Board.

The experience of the G20 might contribute to inject into global health governance an increased macroprudential approach to the management of systemic risks. The example of bottlenecks in essential supply chains shows the importance of a macroprudential approach involving action at the global and national levels, by public and private actors. This could be work involving, among others, the WHO and the WTO.

As we gradually start to reflect upon how to boost preparedness in a post-COVID world, a macroprudential grasp of systemic health risks would better equip global health governance to deal with challenges of this broad, multifaceted nature. It could reveal that coordinated, preventive collaboration might be the remedy to prevent a disease from becoming "too big to heal". 